\title{
Kernos
}

Revue internationale et pluridisciplinaire de religion grecque antique

$24 \mid 2011$

Varia

\section{Joannis MYLONOPOULOS (éd.), Divine Images and Human Imaginations in Ancient Greece and Rome}

\section{Gabriella Pironti}

\section{(2) OpenEdition \\ Journals}

\section{Édition électronique}

URL : http://journals.openedition.org/kernos/1987

DOI : 10.4000/kernos. 1987

ISSN : 2034-7871

\section{Éditeur}

Centre international d'étude de la religion grecque antique

\section{Édition imprimée}

Date de publication : 1 janvier 2011

Pagination : 352-354

ISSN : 0776-3824

Référence électronique

Gabriella Pironti, « Joannis mylonopoulos (éd.), Divine Images and Human Imaginations in Ancient Greece and Rome », Kernos [En ligne], 24 | 2011, mis en ligne le 18 octobre 2011, consulté le 21 septembre 2020. URL : http://journals.openedition.org/kernos/1987 ; DOI : https://doi.org/10.4000/ kernos. 1987 
amis ou élèves, y ont pris part, auxquels il faut ajouter P.B. lui-même, auteur, comme on sait, de ce maitre-ouvrage. Leurs contributions concernent donc exclusivement la religion grecque dans ses composantes féminines; une bibliographie sélective, mais riche, est suivie de plusieurs index. Le second ouvrage, explicitement dédié au père de La fille d'Athènes déborde quelque peu le domaine religieux; il compte une vingtaine d'articles centrés sur trois thèmes principaux entre lesquels apparaissent souvent des recoupements : les femmes, la religion et les corps. Inauguré par une biographie "non autorisée », il se termine par la bibliographie du dédicataire.

Placés sous le signe de l'amitié, les deux ouvrages procèdent d'une même intention : saluer l'homme et l'helléniste en suivant des voies qu'il a lui-même tracées, qu'ils s'agisse des thématiques, comme la religion des femmes, le polythéisme, l'esclavage, ou des démarches, volontiers tournées vers le concret et marquant une prédilection pour la Grèce buissonnière (cf. le c.r. du dernier ouvrage de P.B., La Grèce d'à côté, dans Kernos, 21 [2008], p. 357-359). Sous ce rapport notamment, les deux parcours sont très réussis. Nul ne pourrait se méprendre, en effet, sur l'identité du dieu auquel est voué le kernos d'offrandes que dessinent ces belles études. Ad multos annos, ajouterai-je, en m'associant cordialement à l'hommage rendu.

André Motte (Université de Liège)

Joannis Mylonopoulos (éd.), Divine Images and Human Imaginations in Ancient Greece and Rome, Leiden/Boston, Brill, 2010. 1 vol. 16,5 × 24,5 cm., ill., 437 p., (Religions in the Graeco-Roman World, 170). ISBN : 978-90-04-17930-1.

Issu en grande partie d'un colloque qui s'est déroulé à l'Université d'Erfurt en 20071, ce volume réunit treize études consacrées aux représentations figurées du divin dans les mondes grec (surtout) et romain, et aux différents problèmes que pose aux savants modernes l'interprétation des images des dieux antiques. C'est à signaler ces problèmes que s'attache l'introduction de J. Mylonopoulos, qui ne se limite pas à passer en revue les diverses contributions, mais les intègre dans un riche tissu argumentatif en ouvrant des pistes de réflexion susceptibles de renouveler l'enquête. Organisé suivant un axe à la fois chronologique et thématique, le volume est inauguré par une étude de F. Blakolmer portant sur les images divines dans l’iconographie minoenne et mycénienne; l'A. interroge le décalage problématique entre, d'un côté, le panthéon pleinement articulé dont témoignent les documents écrits et, de l'autre, les représentations figurées des dieux : l'interprétation de ces dernières s'avère extrêmement difficile à cause de la quasi-indistinction entre l'humain et le divin, et de la quasi-absence d'attributs spécifiques, sans oublier les malentendus engendrés par la place prépondérante des images féminines. Ainsi, le déchiffrement du Lineaire $\mathrm{B}$ nous a révélé entre autres choses à quel point nous sommes loin de posséder d'emblée, comme on le croyait autrefois, les clés pour « déchiffrer» l'iconographie de l'âge du Bronze. D'autre part, la neutralité iconique qui s'y trouve privilégiée lorsqu'il est question de mettre en images les dieux, ou bien le roi, est un choix significatif, même si son sens exact reste à découvrir. La place de l'aniconisme dans la représentation du divin en Grèce ancienne fait l'objet de l'étude de M. Gaifman, qui en parcourt les interprétations, de Winckelmann à Nilsson, de Pausanias à Clément d'Alexandrie; concernant la construction culturelle de ce qu'on appelle « aniconisme », l'A. signale à raison la distance entre le point de vue de Pausanias, selon lequel la (prétendue) ancienneté des représentations « aniconiques » se trouvait positivement qualifiée en termes de prestige religieux, et celui des savants modernes qui en jugeant l'aniconisme à l'aune d'un paradigme anthropomorphique et dans une perspective évolutionniste, l'ont disqualifié comme étant «primitif». Le statut de korai archaï-

${ }^{1}$ Le sommaire du volume est donné dans Kernos 23 (2010), p. 415. 
ques est au cœur de la réflexion de C. Keesling : contrairement à ceux qui nient que ces korai aient pu représenter à l'époque archaïque des êtres divins, l'A. signale la possibilité d'une telle identification, l'anonymat apparent pouvant être compensé par l'emplacement de ces images à l'intérieur d'un lieu de culte donné. C'est sur les images archaïsantes des dieux, qui apparaissent dès le Ve siècle av. J.-C., et sur les implications religieuses d'un tel phénomène que se penche F. Hölscher : la représentation, à cette époque, des dieux « à la manière ancienne » n'est pas à mettre en rapport avec une quelconque détérioration du sentiment religieux, mais s'explique, notamment dans l'iconographie des vases, en tant que ressort «narratif», par exemple dans la mise en images contextuelle des statues des dieux et des dieux en action. Pour mieux comprendre le rôle que les statues des dieux peuvent jouer dans le processus de communication avec le divin, V. Pirenne-Delforge choisit des les étudier parallèlement aux prêtres, qui se voient aussi attribuer à l'occasion une fonction analogue à celle de «statues vivantes » et qui jouent surtout un rôle majeur en tant que «intermittent vectors of divine manifestation» (p. 134). En intervenant dans le débat autour de la notion de «statue de culte », l'historienne de la religion grecque se refuse à écarter purement et simplement cette notion et choisit de reformuler la question en signalant le statut spécifique que l'bidrysis, à savoir le rituel qui installe une divinité à un emplacement précis, confère à la représentation iconique ou aniconique de la divinité en question, ne serait-ce que pour un temps.

L'essai de G. Ekroth porte, quant à lui, sur un relief votif ayant trait au culte héroïque de Thésée à Athènes et sur l'identification d'une sorte de pierre basse et arrondie qui apparait au centre de la représentation, entre la figure du héros et celles du dédicant et, sans doute, de son fils; selon l'A., il ne faudrait pas voir dans la pierre en question une variante d'un autel et/ou un autel en particulier, car elle pourrait aussi bien renvoyer à la fois à l'borkomosion, un lieu de la ville ancienne où les serments étaient prêtés, et à un bouclier, dans une construction polysémique évoquant peut-être le serment des éphèbes athéniens en présence de l'éphèbe mythique par excellence. J. Mylonopoulos consacre une étude très fouillée à l'utilisation des attributs dans l'iconographie grecque, dont il met en lumière la sémantique complexe, en rejetant avec raison la thèse des «survivances » et sans en aplatir non plus le sens sur le seul plan dénotatif. Parmi les exemples choisis par l'A. pour illustrer la dynamique de ces signes qui font partie intégrante d'une stratégie visuelle, j’aimerais signaler, à côté de l'image surprenante d'un Ulysse au trident, celle tout aussi curieuse d'une Aphrodite à l'égide sur un vase attique à figures noires. Cet exemple montre bien comment le langage iconographique a développé ses propres moyens pour représenter la polyvalence divine ${ }^{1}$. I. Petrovic analyse, dans son essai, un poème fragmentaire de Callimaque (Iambes VII) où il est question d'une statue d'Hermès Perpheraios, miraculeusement repêchée, et du parcours compliqué qui s'achève avec l'installation d'un culte pour ce dieu; selon l'A., il s'agit moins d'un véritable aition que d'une allégorie où la statue archaïsante du dieu représenterait l'ancienne poésie iambique, à la fois simple et vénérable, venue réclamer sa part d'honneur à l'époque hellénistique. L'étude de T. Scheer s'articule en deux volets : dans le premier, l'A. s'interroge sur les raisons qui poussent Auguste à soustraire aux habitants de Tégée l'ancienne statue d'Athéna Aléa, sans doute non seulement pour punir ces Arcadiens qui avaient pris parti pour Antoine lors de la bataille d'Actium, mais peut-être aussi pour installer à Rome une image «mythiquement » chargée, évoquant à la fois les ancêtres arcadiens des Romains et la chasse de Calydon. Dans le second volet, elle analyse la réaction des Tégéates qui n'ont aucun problème à remplacer cette vénérable image. Néanmoins, ils choisissent pour ce faire non pas une image d'Athéna Aléa provenant d'une autre cité, mais celle d'une Athéna Hippia provenant des environs de la leur. Ce choix significatif montre d'un côté que la puissance divine d'Athéna Aléa peut changer de «siège» pour se manifester, confirmant ainsi

\footnotetext{
${ }^{1}$ Cf. G. Pironti, « Aphrodite à l'égide ou de la distraction des peintres », Mètis, n.s. 8 (2010), p. 255-275.
} 
que le rapport d'identification entre une divinité et son image est relatif, et de l'autre que l'enracinement territorial d'un culte est un facteur déterminant dans la communication avec le divin.

Les études restantes concernent le rapport entre religion et représentations figurées à la période impériale et dans le monde romain. En analysant l'emplacement des statues des empereurs romains dans les temples des dieux grecs et leurs spécificités iconographiques, D. Steuernagel affirme que les empereurs synnaoi ne seraient pas hiérarchiquement subordonnés aux dieux traditionnels et se verraient confier par les communautés locales la fonction d'intermédiaires privilégiés dans la communication avec ces derniers. Une telle fonction est reconnue également par S. Estienne qui ne renonce toutefois pas à signaler les hiérarchies dynamiques se dessinant à l'intérieur des temples en relation avec l'identité du dédicataire attitré; l'A. livre aussi, dans la première partie de son étude, une analyse du vocabulaire romain en matière d'images divines (signum, simulacra deorum), soulignant le statut juridique particulier des ornamenta aedium, qui ne seraient pas inaliénables. C'est sur le rôle des simulacra deorum dans la fondation même d'un sanctuaire que s'interroge K. Moede qui, à l'appui de certains reliefs romains représentant l'installation de statues à la présence d'un autel et des autorités religieuses, propose d'y reconnaitre une formule visuelle traduisant en image la procédure rituelle d'introduction de nouveaux cultes. C'est à la réception des images des dieux anciens que s'attache enfin A. Bravi dans un essai consacré à l'époque byzantine : pendant longtemps, les statues antiques installées à Constantinople ne sont pas que décoratives au sens moderne du terme, mais continuent d'agir en tant que vecteurs identitaires et symboles de pouvoir.

L'ouvrage est complété par de précieux indices et par de tout aussi précieuses illustrations qui permettent de suivre aisément les démonstrations des différents auteurs. Mais l'éditeur du volume est à remercier avant tout pour l'introduction qu'il a livrée et dont la lecture s'impose tant avant qu'après avoir parcouru les arguments déployés dans les études qui composent l'ensemble. On y trouve en effet une réflexion de fond sur les points cruciaux soulevés par cet ouvrage, à partir de la relation problématique entre « divine images » et « cult images ». De cette stimulante lecture, on retiendra une invitation à appréhender les spécificités du langage iconographique quand il «parle » de religion, mais aussi à replacer les différentes représentations du divin dans leurs contextes cultuels, rituels et historiques, et enfin à croiser les données émiques avec les outils heuristiques offerts par une approche "étique », dans le but de comprendre depuis notre perspective éloignée comment le polythéisme se traduisait autrefois en images.

\section{Gabriella Pironti (Università degli Studi di Napoli 'Federico II' Centre ANHiMA [Paris])}

\section{Thomas MORARD, Horizontalité et verticalité. Le bandeau humain et le bandeau divin chez le Peintre de Darius, Mainz, von Zabern, 2009. 1 vol. $21,5 \times 30 \mathrm{~cm}, \mathrm{X}+$ 264 p., 120 pl. ISBN : 978-8053-3965-0}

Cet ouvrage est issu d'une thèse soutenue en 2008 à l'Université Lumière - Lyon 2, sous la direction de Jean-Marc Moret. L'étude porte sur un groupe de vases particuliers, à savoir des pièces apuliennes dont le décor est divisé en deux frises superposées : la frise supérieure est réservée aux divinités et la frise inférieure aux humains. Bien que ces vases aient très tôt suscité l'attention, aucune étude systématique n'avait encore été consacrée à ce type de représentation, ce que propose de faire l'A. Contrairement à ce que pourrait laisser entendre le sous-titre, l'ouvrage n'est pas consacré uniquement au Peintre de Darius, bien que les réalisations de ce dernier y occupent une place importante. C'est lui qui semble avoir donné une forme canonique à ce type de composition, en faisant du double registre sa spécialité. 